\title{
Probabilistic Financial Decision Support Framework
}

\author{
Chiu-Che Tseng \\ Dept. of Computer Science and Information Systems \\ Texas A\&M University-Commerce
}

\begin{abstract}
The challenge of the business decision support domain is that a large amount of diverse information can be potentially relevant to a decision, and that, frequently, the decisions have to be made in a timely manner. This presents the potential for better decision support, but poses the challenge of building a decision support system for timely decision support.

These problems motivate us to investigate ways in which the decision maker can be equipped with a flexible real time decision support system to be practical in time-critical situations. For this purpose, we propose a system that uses the Object Oriented Bayesian Knowledge Base (OOBKB) design to create a decision model at the most suitable level of detail to produce an decision recommendation within a reasonable length of time. The decision models our system uses are implemented as influence diagrams.

We validate our system with experiments in a simplified investment domain. The experiments show that our system produces a quality recommendation under different situations.
\end{abstract}

\section{Introduction}

The goal of a decision support system is to provide the human user an optimized decision recommendation when operating under uncertainty in complex environments. The particular focus of our discussion is the investment domain investment decision-making is to select an optimal portfolio that satisfies the investor's objective, or, in other words, to maximize the investment returns under the constraints given by investors. The investment domain contains numerous and diverse information sources, such as expert opinions, news releases and economic figures, etc. This presents the potential for better decision support, but poses the challenge of building a decision support agent for accessing, filtering, evaluating, incorporating information from different sources, and for making final investment recommendations. Time is frequently an important factor in the investment domain. It is sometimes impossible for an investor to utilize all the available information and come up with a decision in a timely fashion. Therefore, a control mechanism is needed to help the agent balance between deliberation and timely decisionmaking.

These problems motivate us to investigate ways for equipping our system with flexible real time decision support mechanisms to be applicable in time-critical situations. The real time decision support considers the tradeoff between the decision support quality versus the computational time and cost. In other words, the goal of our system is to provide the investor with the best decision support under time constraints. For this purpose, we propose a system that uses the Object Oriented Bayesian Knowledge Base (OOBKB) design to create a decision model at the most suitable level of detail to produce an investment recommendation within a reasonable length of time. The more detailed models, located at the bottom of the class hierarchy, are more time consuming to work with and may require more information gathering, but provide better quality of decision support. The more abstract models, located higher in the class hierarchy, can be evaluated faster but result in less precise recommendations. We allow the decision maker to decide which detail level of recommendation that he or she requires based on their assessment of the situation. By using our proposed real time decision support and information gathering system, the investor will have a flexible real time decision support system for integrating the external information and producing a viable investment decision recommendation.

\section{Related Work}


We explored the way to reduce the complexity of the investment decision deliberation that might cause the investor to lose money under urgent situations, and, at the same time, to provide the highest quality investment recommendations possible.

There are various research on building and developing decision support system for business. The Noori and Salimi [7] proposed a decision support system use in the business to business marketing domain. Their system focus on the business to business aspect. Lari [5] proposed a decision support system that utilized case based reasoning on quality issues. Rosca, Greenspan and Wild [9] proposed a system that help automated the business rules lifecycle.

\section{Probabilistic Framework for Decision Support}

To represent the domain knowledge we use an object-based system. Here objects are used to represent domain information and decisions, and to organize the domain knowledge using the objectoriented paradigm into a hierarchy of concepts and classes. In order to construct a well-defined knowledge base system, we adopt an ontological hierarchy of concepts. This class hierarchy organizes and simplifies the knowledge base through inheritance. Figure 3 depicts the highest level of the ontology. The root class, Things, consists of two subclasses, abstract and physical objects. Each of these subclasses consists of some other subclasses. The subclass relationship organizes the classes into a taxonomic hierarchy.

There is a limitation in the traditional knowledge base representation, in that there is no probability information available to represent uncertainty. For example, a car class contains an attribute representing the color of the car. If the color is unknown, in the traditional knowledge base the attribute will simply be encoded as unknown. We would like to have this attribute represented as a range of values and associate it with a probability distribution in order to reason under uncertainty. Koller and Pfeffer [4] described the concept of constructing the Bayesian network from several subnets that represent different objects in the domain. Yelland [14] organized the knowledge base into a collection of objects that represent the domain knowledge. For this purpose, we applied the concepts from Koller and Pfeffer, and Yelland into an object oriented Bayesian knowledge base.

The Object-Oriented Bayesian Knowledge Base (OOBKB) is the heart of our system-it stores and organizes the domain information. The domain information in the OOBKB is organized into a hierarchy of classes, which represents the generalization and specialization of the concepts in our domain. Since some of the values of the attributes of the instantiations of classes are not known with certainty, we use them as chance nodes in an influence diagram. Thus, the OOBKB contains the probability and casual information, from which we can derive and create influence diagrams on the fly. Since our OOBKB organizes the classes in a hierarchical order, we are able to create influence diagrams on different levels. The different levels of instantiation represent the decision models from abstract to detailed. The more detailed the decision model, the more nodes are explicitly represented within the influence diagram.

The OOBKB can be created and updated offline to provide an up to date representation of the domain. This can take the computational burden out of runtime, thus increasing the performance of our system. The learning process can include updating the conditional probability tables (CPTs) and prior distributions in each class. In our financial domain, we created the knowledge base from consulting with the domain experts and our probability tables are generated from the historic data we gathered from the Compustat database.

\subsection{Investment Decision Support Knowledge Base}

There are more than 2000 stocks to pick from in today's market. It would be computationally infeasible to create a huge network that incorporates all the companies and the information sources in order to provide the investor with a portfolio recommendation. To handle the complexity issue, we created a hierarchical Object Oriented Bayesian Knowledge Base (OOBKB) for the investment domain.

The Object Oriented Bayesian Knowledge Base (OOBKB) is the center of our system - it stores and organizes the domain information. The domain information in the OOBKB is organized into a hierarchy of classes, which represents the generalization and specialization of the concepts in 
our domain. In figure 1 we illustrated the object oriented Bayesian knowledge base that represents a simple investment domain. Since some of the values of the attributes of the instantiations of classes are not known with certainty, we use them as chance nodes in an influence diagram. Thus, the OOBKB contains the probability and casual information, from which we can derive and create an influence diagram on the fly. For example in figure 2, the bank sector class contains an attribute called bank_MBValue representing the market to book value financial ratio of the bank sector stocks within the S\&P 500. The bank_MBValue has a type definition of $t$ Simpleslot class. The $\mathrm{t}$ SimpleSlot class is used to represent a chance node definition, so by assigning the bank_MBValue attribute to t_SimpleSlot class type we defined the bank_MBValue as a chance node in our knowledge base. Since our OOBKB organizes the classes in a hierarchical order, we are able to create influence diagrams on different levels. The different level of instantiation represents the decision model from abstract to detailed. In the investment domain, the more detail the decision model, the more investment factors will be represented within the influence diagram.

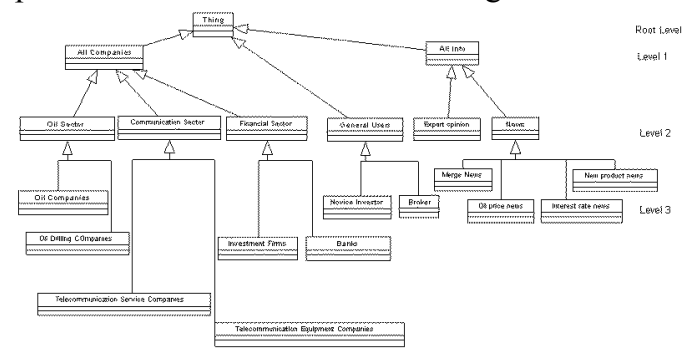

Fig. 1: The class hierarchy of the OOBKB in a simple investment domain

The level of detail for the decision model is controlled by the urgency factor. The more urgent the invest situation is the less detailed the decision model is generated.

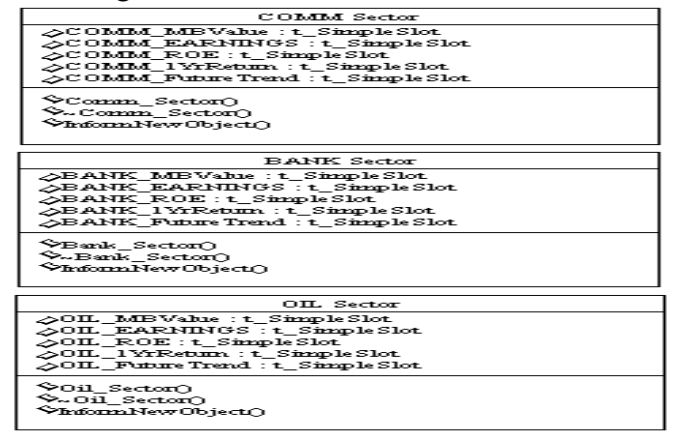

Fig. 2: The classes instantiated detail from OOBKB
Figure 3 illustrates the influence diagram that our system creates from the object oriented Bayesian knowledge base. The influence diagram that the system created is from the level two classes that represent a decision model for selecting among different sectors of the stocks. The abstract decision model gives advice to the investor at a coarse level; it will recommend one or more sectors of stocks to the investor. Our system generates this type of decision model only when there is an urgent situation that will not allow the system to spend more time on the computation.

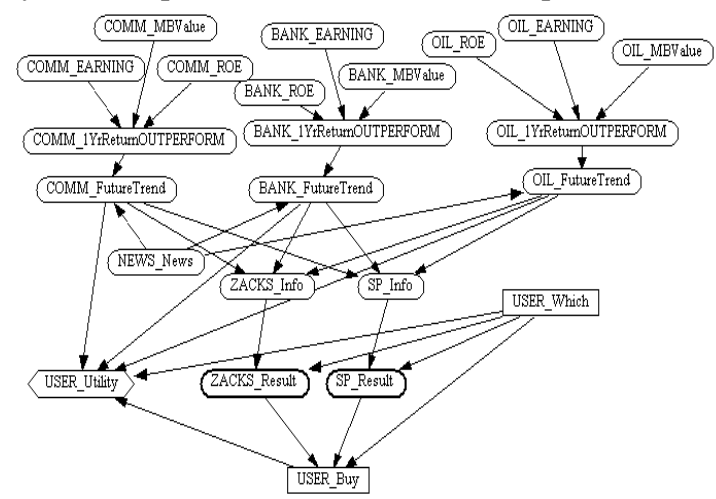

Fig. 3: Decision model created from level two classes.

\section{Experiment}

The experiments in this section are designed to validate how the real-time decision support and information gathering concept can be realized by actually applying the system to the investment domain.

We put our system in a simulated investment situation and assess the performance when real time decision support and information gathering procedures are employed.

Our model of the investment domain consists of a number of stocks for the investor to construct an investment portfolio. The goal is to maximize the profit from the investment portfolio. The experiments are written in $\mathrm{C}++$ and built on top of the Netica Belief network package, running on a LINUX platform.

\subsection{Experiment Results}

We tested our system on actual stock market data we collected from Compustat database for the period of the first quarter 2001. For experimental runs, we selected 12 companies from SP500 company listing. We divided the companies into 
three sectors, communication, banking, and oil production sector.

Our system selected the detailed decision model, as in figure 9, returned the recommendations of getting external information from Zacks investment Inc. for all but one company, and returned the recommendations of buying 7 out of 12 available companies.

We track the one-day performance of our portfolio assuming there were no changes in the external information nodes that will affect the decision recommendations of our system. The portfolio that our system constructed ended up with the cash value of $\$ 8,661.19$ at the closing of Jun 5 , 2001 , a $0.4 \%$ increase for the day.

\section{Conclusions}

We have presented a framework for using an Object Oriented Bayesian Knowledge Base to aid the investor in a time critical situation. In our approach, the agent's knowledge is represented as an influence diagram created from the different levels of the OOBKB. The agent can use this model to gather extra information and make decision recommendations to the investor.

There are still some issues we would like to research further. The ontology for the knowledge base is constructed by hand, we would like to research an automate method for creating the structure by learning from the business data. And we would also like to incorporate automate information gathering capability to update the data contains in the knowledge base so it can be up to date on the current development of the business situation.

Finally, we expect that we will be able to develop a more comprehensive system through ongoing work on the above research issues and applying our framework to various domains.

\section{REFERENCES}

[1] Binder, J., Koller, D., Russell, S., Kanazawa, K., 1997, Adaptive Probabilistic Networks with Hidden Variables. Machine Learning, vol. 29, pp. 213-244, 1997.

[2] G. Cooper, and E. Herskovits. A Bayesian method for the induction of probabilistic networks from data. Machine Learning 9, pp. 309-347, 1992.

[3] F. V. Jensen. An Introduction to Bayesian Networks. New York, NY: Springer-Verlag, 1996.
[4] D. Koller and A. Pfeffer. Object-Oriented Bayesian Networks. In Proceedings of the 13th Annual Conference on Uncertainty in Artificial Intelligence, pp. 302-313, August 1997.

[5] Lari. A decision support system for solving quality problems using case-based reasoning. Total Quality Management and Business Excellence 14, no. 6, pp. 733-745, 2003.

[6] M. L. Mitchell and J. H. Mulherin. The Impact of Public Information on the Stock Market. Journal of Finance, vol. 49, issue 3, pp. 923950, 1994.

[7] B. Noori and M. H. Salimi. A decision-support system for business-to-business marketing. The Journal of Business and Industrial Marketing, no. 4/5, pp 226-236, 2005.

[8] D. K. Pfeffer, B. Milch, and K. Takusagawa. SPOOK: A system for probabilistic objectoriented knowledge representation. In Proceedings of the 15th Annual Conference on Uncertainty in AI (UAI), Stockholm, Sweden, pp. 541-550, August 1999.

[9] D. Rosca, S. Greenspan and C. Wild. Enterprise Modeling and Decision-Support for Automating the Business Rules Lifecycle, Automated Software Engineering 9, no. 4, pp. 361-404, 2002.

[10]S. J. Russell and P. Norvig. Artificial Intelligence, A Modern Approach. EngleWood Cliffs, New Jersey: Prentice Hall, 1995.

[11]C. Tseng and P. J. Gmytrasiewicz. A Real Time Investment Decision Support System. In Proceeding of the International Conference on Artificial Intelligence and Soft Computing, May 2001.

[12] S. R. Watson and R. V. Brown. The valuation of decision analysis. Journal of the Royal Statistical Society, 141, Part I, pp. 69-78, 1978.

[13]M. P. Wellman, J. S. Breese, and R. P. Goldman. From knowledge bases to decision models. The Knowledge Engineering Review, 7(1):pp. 35-53, 1992.

[14] M. P. Yelland. Object oriented knowledge representation. BT Technology Journal, 11(3):pp. 41-51, July 1993. 